\title{
PANCREATECTOMIA DISTAL VIDEOLAPAROSCÓPICA EM PACIENTES COM CISTADENOMA DE PÂNCREAS
}

\author{
Marcel Autran C. MACHADO, Leonardo F. CANEDO, Paulo HERMAN, \\ André L. MONTAGNINI, Rubens A. A. SALLUM e Marcel Cerqueira Cesar MACHADO
}

RESUMO - Racional - O diagnóstico de tumores císticos do pâncreas vem aumentando nos últimos anos. Estes tumores acometem geralmente pacientes do sexo feminino e apresentam poucos sintomas. Sua remoção por videolaparoscopia está indicada em pacientes selecionadas, principalmente quando localizados na região distal do pâncreas. Objetivos - É apresentada a experiência inicial de um grupo de cirurgiões na realização de pancreatectomia distal por videolaparoscopia em pacientes com cistadenoma pancreático. Material e Métodos - Três pacientes do sexo feminino (idade média, 55 anos) foram submetidas a ressecção pancreática por videolaparoscopia entre setembro de 2001 e dezembro de 2003. Resultados - A ressecção pancreática por videolaparoscopia foi realizada com sucesso nas três doentes. O tempo cirúrgico variou de 4 a 6 horas. O sangramento operatório foi mínimo em todos os casos. A aplicação do grampeador endoscópico foi difícil em uma paciente devido à espessura do pâncreas. As três pacientes evoluíram bem, recebendo alta entre o 2 e e o 5 o dia pós-operatório. Duas apresentaram fístula pancreática com resolução após tratamento conservador. Conclusões - A pancreatectomia laparoscópica é factível, pode trazer benefícios aos pacientes portadores de neoplasia cística da porção distal do pâncreas, com pouca dor pós-operatória, curto tempo de permanência hospitalar, baixo índice de complicações e melhor resultado estético.

DESCRITORES - Pancreatectomia. Neoplasias pancreáticas, cirurgia. Cistadenoma. Laparoscopia.

\section{INTRODUÇÃO}

A videolaparoscopia foi um dos avanços mais importantes da cirurgia do aparelho digestivo nos últimos anos. O desenvolvimento do instrumental laparoscópico e o aprimoramento técnico obtido com o acúmulo de experiência, possibilitaram a realização de cirurgias consideradas de alta complexidade pela técnica minimamente invasiva no mundo todo e também no nosso meio ${ }^{(5,7,8)}$.

O uso da laparoscopia para afecções do pâncreas foi utilizada inicialmente para o estadiamento de doenças neoplásicas ${ }^{(13)}$, tratamento paliativo de sintomas obstrutivos ${ }^{(5,8,13)}$ ou drenagem de pseudocistos ${ }^{(13)}$. Porém, apenas recentemente, as ressecções pancreáticas laparoscópicas passaram a ser realizadas em pacientes selecionados ${ }^{(2,3)}$. A dificuldade técnica, o baixo número de pacientes elegíveis para a cirurgia, além do questionamento quanto à radicalidade oncológica em doenças malignas, não possibilitaram o estabelecimento da pancreatectomia laparoscópica como procedimento padrão.

Apesar disso, a pancreatectomia distal laparoscópica tem sido muito utilizada no tratamento de tumores benignos e pseudocistos intra-parenquimatosos ${ }^{(10)}$. A anatomia local mais favorável, quando comparada às porções mais proximais do pâncreas, e a ausência de anastomoses e da necessidade de linfadenectomia, favorece esta abordagem ${ }^{(2,3,12,15)}$.

Neste estudo, apresenta-se experiência inicial com pancreatectomia distal por videolaparoscopia, utilizada na ressecção de cistadenomas pancreáticos.

\section{CASUÍSTICA}

Todos os pacientes submetidos a ressecção pancreática por videolaparoscopia no Hospital do Câncer de São Paulo no período de setembro de 2001 a dezembro de 2003, foram incluídos neste estudo.

Pacientes com tumores malignos de pâncreas ou tumores localizados na cabeça e corpo proximal não eram considerados para ressecção por videolaparoscopia por motivos oncológicos e técnicos. Pacientes com tumor de pâncreas submetidos a laparoscopia para estadiamento ou intervenção paliativa não foram objeto do presente estudo.

As três pacientes desta casuística apresentavam neoplasia cística do pâncreas sem sinais de malignização e com localização distal no pâncreas (Figura 1) sendo, desta maneira, doentes selecionados para o procedimento videolaparoscópico.

Trabalho realizado no Departamento de Cirurgia Abdominal do Hospital do Câncer de São Paulo, SP.

Endereço para correspondência: Dr. Marcel Autran Cesar Machado - Rua Evangelista Rodrigues, 407 - 05463-000 - São Paulo, SP. E-mail: dr@drmarcel.com.br 


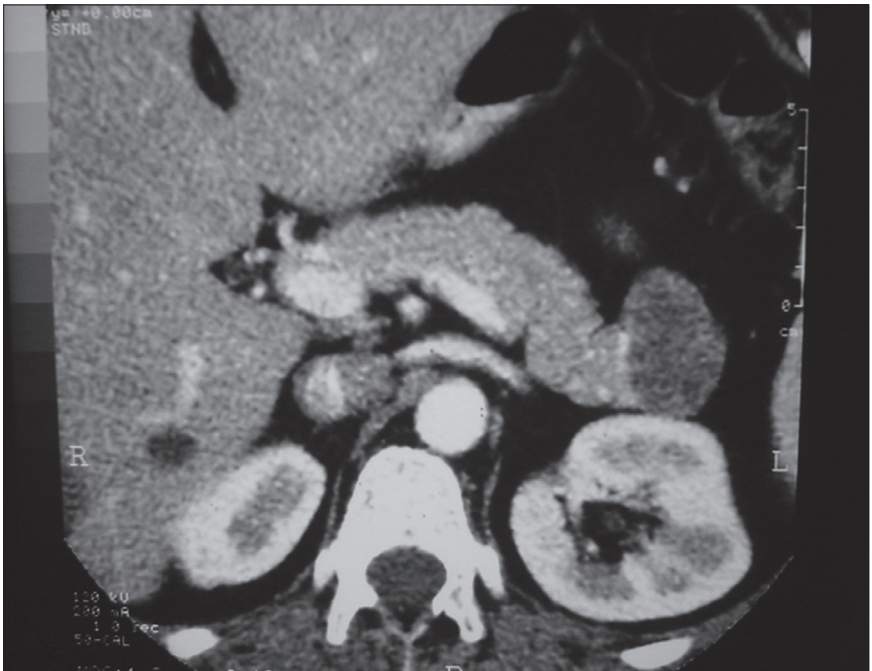

FIGURA 1 - Tomografia computadorizada de abdome. Note tumor cístico na cauda do pâncreas

\section{TÉCNICA}

Sob anestesia geral, com a paciente em decúbito dorsal horizontal, foi realizada incisão supra-umbilical, colocação de trocarte de $10 \mathrm{~mm}$ sob visão direta ${ }^{(6)}$ e instalação de pneumoperitônio. Três outros portos, em flanco esquerdo $(5 \mathrm{~mm})$, pararretal esquerdo $(10 \mathrm{~mm})$ e epigástrico (5 mm) foram instalados (Figura 2). Em uma das pacientes foi necessário o uso de porto auxiliar de $5 \mathrm{~mm}$ em região pararretal direita. $\mathrm{O}$ cirurgião postou-se à direita do paciente, com o primeiro auxiliar em frente e o segundo auxiliar (câmera) à sua direita.

A intervenção iniciou-se com abertura da retrocavidade dos epíploons após ligadura e secção dos vasos da grande curvatura gástrica, utilizando o bisturi harmônico. Neste tempo, foi realizada a inspeção do pâncreas, avaliando-se a textura, a coloração e a lesão a ser ressecada. A artéria esplênica foi dissecada no bordo superior do pâncreas e foi realizada a ligadura perdida da mesma meneira da aberta. Realizou-se a liberação do polo inferior e superior do baço, seguido de introdução de afastador atraumático triangular que ajuda na exposição do hilo esplênico ${ }^{(9)}$. Uma vez liberado o baço, prossegue-se com a dissecção do retroperitônio e liberação do pâncreas. A artéria esplênica é, então, seccionada junto ao local da secção do pâncreas com margem de segurança em relação ao tumor pancreático. O pâncreas é então seccionado com grampeador linear endoscópico (Figura 3). O coto pancreático é examinado para possíveis locais de sangramento e, se necessário, são dados pontos hemostáticos em $\mathrm{X}$ com fio inabsorvível. As lojas esplênica e pancreática são drenadas por meio de dois drenos de Penrose largos, exteriorizados pelo porto $(10 \mathrm{~mm})$ do flanco esquerdo. A peça é colocada em saco plástico resistente. A boca do saco plástico é exteriorizada através do porto umbilical. Com o saco aberto, o pâncreas contendo o cistadenoma é separado do baço e retirado inteiro para análise histopatológica e o baço é macerado.

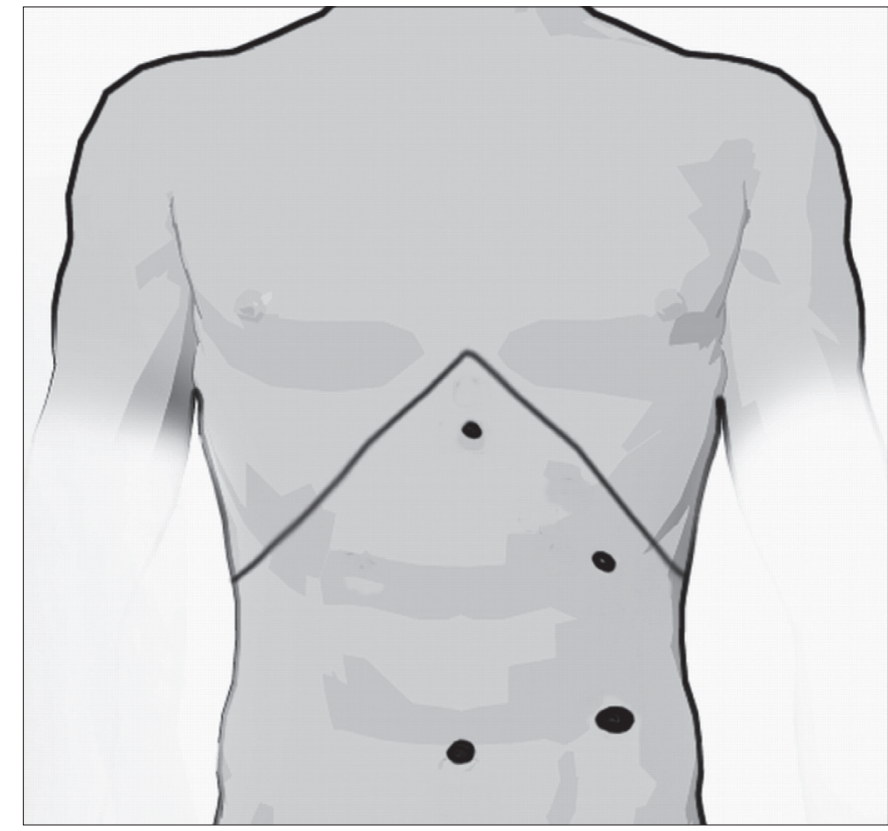

FIGURA 2 - Desenho esquemático mostrando a colocação dos trocartes para a realização de pancreatectomia distal por videolaparoscopia

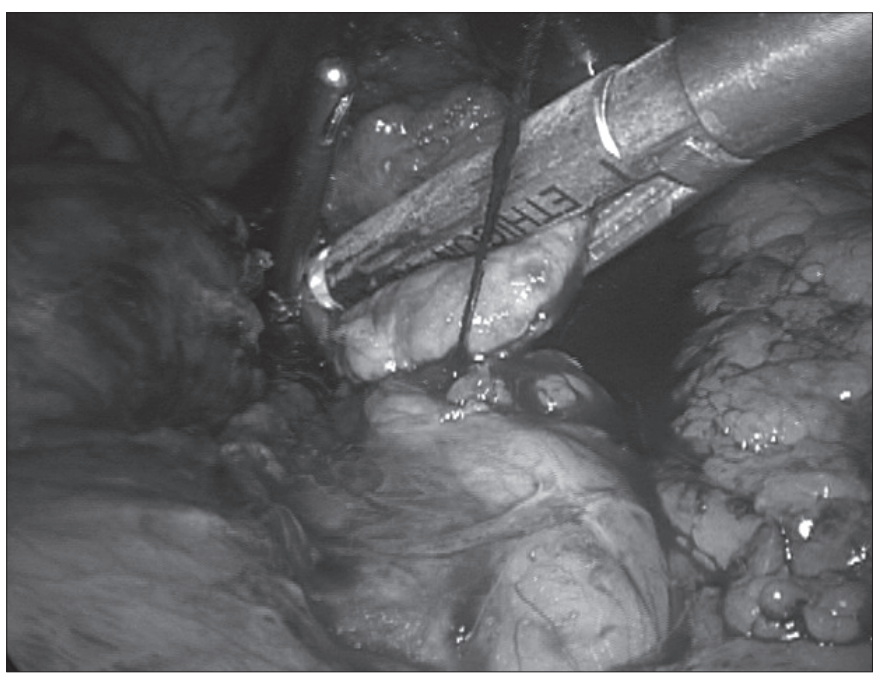

FIGURA 3 - Fotografia do intra-oparatório. O pâncreas está sendo tracionado para cima, enquanto o grampeador endoscópio com carga vascular é aplicado

\section{RESULTADOS}

A ressecção pancreática por videolaparoscopia foi realizada com sucesso em três doentes, todas do sexo feminino, portadoras de cistadenoma do pâncreas. A idade média foi de 55 anos (variação: 51 a 68). Duas pacientes apresentavam dor abdominal não-característica e uma doente era assintomática, sendo o cistadenoma achado incidental de exame de imagem. O tamanho do cistadenoma variou de 2 a $4 \mathrm{~cm}$. Em todas as pacientes o diagnóstico definitivo foi feito através de tomografia computadorizada (Figura 1). Em nenhuma paciente foi observada 
calcificação central, característica de cistadenoma seroso. A indicação cirúrgica em dois casos foi a impossibilidade de diagnóstico diferencial entre cistadenoma seroso e mucinoso. A paciente mais velha da casuística foi submetida, em outro serviço, a ultra-sonografia endoscópica e punção do cistadenoma que foi compatível com cistadenoma mucinoso. Em nenhum caso o baço foi preservado pois, em todos eles, o tumor cístico estava localizado junto ao hilo esplênico. Em uma das pacientes foi encontrado um baço acessório que foi preservado com sucesso. Em nenhum dos casos foi realizado auto-implante esplênico.

O tempo cirúrgico variou de 4 a 6 horas, sendo maior na primeira paciente. Em uma das pacientes, a retirada da peça foi feita através de incisão transversa sem maceração do baço, que era muito pequeno. O sangramento operatório foi mínimo em todos os casos. Não houve necessidade de transfusão sangüínea em nenhuma paciente no intra-operatório ou no período pósoperatório. Em todos os casos houve necessidade de sutura do coto pancreático para completa hemostasia. A aplicação do grampeador endoscópico foi difícil em uma paciente devido à espessura do pâncreas.

O exame anatomopatológico foi cistadenoma seroso em duas e mucinoso em uma paciente, confirmando o achado préoperatório.

As três pacientes evoluíram bem, recebendo alta entre o $2^{\circ}$ e $5^{\circ}$ dia de pós-operatório. Em todas o dreno foi deixado por período mínimo de 10 dias com dosagem seriada de amilase. Duas pacientes apresentaram fístula pancreática com resolução espontânea com tratamento conservador. Uma paciente (68 anos) apresentava pâncreas atrófico e não evoluiu com fístula pancreática no pós-operatório. $\mathrm{O}$ aspecto estético final foi excelente em todos os casos. Todas as pacientes estão bem, sem complicação no período pós-operatório tardio, com seguimento médio de 27 meses.

\section{DISCUSSÃO}

O diagnóstico de tumores císticos do pâncreas vem aumentando nos últimos anos. Isso é conseqüência, principalmente, do aperfeiçoamento e uso mais freqüente de exames de imagem $^{(11)}$. BALCOM et al. ${ }^{(1)}$, em revisão da experiência com ressecções pancreáticas do Massachusetts General Hospital, observaram aumento no número de ressecções pancreáticas por tumores císticos no decorrer da década de 1990.

Os tumores císticos mais freqüentes são classificados em serosos e mucinosos. Os cistadenomas serosos são tumores bem delimitados, que consistem de múltiplos cistos pequenos e são, geralmente, menores que $2 \mathrm{~cm}$ de diâmetro. Esses tumores não têm potencial de malignização. Já os cistadenomas mucinosos, são lesões normalmente multi-loculares e apresentam na sua superfície interna, projeções papilares. São tumores pré-malignos e por esse motivo, devem ser sempre ressecados. O diagnóstico diferencial através dos exames de imagem é muitas vezes difícil e, não raro, realizado apenas após exame anatomopatológico.

Normalmente, a realização de pancreatectomia distal convencional necessita incisões subcostais amplas para se obter uma abordagem segura. A localização retroperitonial do pâncreas e sua íntima relação com estruturas vasculares importantes dificultam a sua exposição. Essas incisões ampliadas estão associadas à dor mais intensa no pós-operatório, além de maior incidência de infecção de ferida operatória e hérnia incisional, se comparado às técnicas minimamente invasivas.

A videolaparoscopia representou um grande avanço para cirurgia do aparelho digestório. Muitas mudanças ocorreram desde a experiência inicial no começo da década de 1990 até os dias atuais. Procedimentos antes realizados através de incisões amplas são hoje realizados pela via laparoscópica de forma segura, com menor morbidade e menor tempo de recuperação pós-operatória. No entanto, apesar do ímpeto de alguns profissionais, o pâncreas sempre exerceu certo temor nos cirurgiões.

A história de ressecções pancreáticas por laparoscopia é relativamente recente. GAGNER e POMP(3) descreveram sua experiência inicial em 1994 e desde então, surgiram relatos de pancreatectomia distal realizadas em diferentes partes do mundo ${ }^{(1,2,4,14)}$. A experiência ainda é muito limitada mas, mesmo assim, alguns trabalhos apontam que a laparoscopia pode ter grande utilidade nas ressecções de tumores císticos distais do pâncreas ${ }^{(10,11)}$. Os estudos ressaltam a natureza benigna das lesões, o que dispensa a realização de linfadenectomia e a localização distal favorável à ressecção, uma vez que não é necessária a realização de anastomoses. Além disso, em se tratando de tumores que acometem mais freqüentemente mulheres e na faixa etária dos 40-50 anos, a questão estética não pode ser negligenciada.

Em trabalho recente ${ }^{(10)}$ com grande número de pacientes, os autores concluíram que essa intervenção pode ser realizada com segurança por cirurgiões experientes, sem mortalidade e com baixa morbidade. Provavelmente, os pacientes portadores de tumores císticos podem se beneficiar, na realização de pancreatectomia distal, de um pós-operatório mais rápido e com menos complicações relacionadas à incisão. Com efeito, na experiência inicial os pacientes desta série apresentaram tempo de internação relativamente curto, bom efeito cosmético, dor pós-operatória de fácil controle e com morbidade equivalente à intervenção convencional.

A fístula pancreática continua sendo uma das principais complicações pós-operatórias da pancreatectomia distal seja por via convencional, ou laparoscópica. No entanto, sabe-se que a fístula pancreática é muito freqüente e para o diagnóstico é necessária a dosagem da amilase do líquido do dreno, que não é realizada em todos os grupos. O aparecimento de fístula pancreática em duas das três pacientes operadas mostra a alta freqüência desta complicação após ressecção pancreática. O uso de grampeadores mecânicos para secção do parênquima pancreático facilita a realização desta intervenção por via laparoscópica.

Conclui-se que a pancreatectomia laparoscópica é factível, pode trazer benefícios aos pacientes com neoplasia cística da porção distal do pâncreas, com pouca dor pós-operatória, curto tempo de permanência hospitalar, baixo índice de complicações e melhor resultado estético. Estudos controlados e com número maior de pacientes são necessários para a confirmação dos achados do presente trabalho. 
Machado MAC, Canedo LF, Herman P, Montagnini AL, Sallum RAA, Machado MCC. Laparoscopic distal pancreatectomy for pancreatic cystadenomas. Arq Gastroenterol 2005;42(3):157-60.

ABSTRACT - Background - Cystic neoplasms are an uncommon group among pancreatic tumors. These lesions are seen more frequently in recent surgical practice, probably because of advances in diagnostic and surgical techniques. Total tumor resection provides the best chance of cure and may remove the risk of malignant transformation of the cystadenomas, particularly of the mucinous type. Minimally invasive techniques have been revolutionary and provide clinical evidence of decreased morbidity and comparable efficacy to traditional, open surgery. However, laparoscopic pancreatic resection is not an established treatment for tumors of the pancreas. Aim - The authors present their initial experience with laparoscopic distal pancreatectomy for pancreatic cystadenomas. Material and Methods - Three female patients (mean age, 55 years) underwent laparoscopic pancreatic resection between September 2001 and December 2003. Results - Laparoscopic pancreatic resection was successfully performed in all patients. Operative time varied between 4 and 6 hours. Intraoperative bleeding was minimal. Due to a thick pancreas, the application of vascular endoscopic stapler was difficult in one patient. Two patients presented postoperative pancreatic leakage with spontaneous resolution. Conclusions - Resection of the pancreas can be safely performed via the laparoscopic approach with all the potential benefits to the patients of minimally invasive surgery.

HEADINGS - Pancreatectomy. Pancreatic neoplasms, surgery. Cystadenoma. Laparoscopy.

\section{REFERÊNCIAS BIBLIOGRÁFICAS}

1. Balcom JH, Rattner DW, Warshaw AL, Chang Y, Fernandez-del Castillo C. Ten-year experience with 733 pancreatic resections: changing indications, older patients, and decreasing length of hospitalization. Arch Surg 2001;136:391-8.

2. Cuschieri A, Jakimowicz JJ, van Spreeuwel J. Laparoscopic distal $70 \%$ pancreatectomy and splenectomy for chronic pancreatitis. Ann Surg 1996;223:280-5.

3. Gagner M, Pomp A. Laparoscopic pylorus-preserving pancreatoduodenectomy. Surg Endosc 1994;8:408-10.

4. Grace PA, Williamson RC. Modern management of pancreatic pseudocysts. Br J Surg 1993;80:573-81.

5. Machado MA, Rocha JR, Bove C, Machado MC. Laparoscopic treatment of duodenal obstruction in a patient with pancreatic cancer. Rev Hosp Clin Fac Med São Paulo 1997;52:35-7.

6. Machado MA, Rocha JR, Machado MC. An alternative technique for open laparoscopy Rev Hosp Clin Fac Med São Paulo 1998;53:174-5.

7. Machado MA, Herman P, Rocha JR, Machado MC. Primary intrahepatic lithiasis: report of a case treated by laparoscopic bilioenteric anastomosis. Surg Laparosc Endosc Percutan Tech 1999;9:207-10.

8. Machado MA, Rocha JR, Herman P, Montagnini AL, Machado MC. Alternative technique of laparoscopic hepaticojejunostomy for advanced pancreatic head cancer. Surg Laparosc Endosc Percutan Tech 2000;10:174-7.

9. Machado MA, Makdissi FF, Herman P, Montagnini AL, Sallum RA, Machado MC Exposure of splenic hilum increases safety of laparoscopic splenectomy. Surg Laparosc Endosc Percutan Tech 2004;14:23-5.

10. Park AE, Heniford BT. Therapeutic laparoscopy of the pancreas. Ann Surg 2002;236:149-58

11. Sheehan MK, Beck K, Pickleman J, Aranha GV. Spectrum of cystic neoplasms of the pancreas and their surgical management. Arch Surg 2003;138:657-60.
12. Sussman LA, Christie R, Whittle DE. Laparoscopic excision of distal pancreas including insulinoma. Aust N Z J Surg 1996;66:414-6.

13. Targarona EM, Pera M, Martinez J, Balague C, Trias M. Laparoscopic treatment of pancreatic disorders: diagnosis and staging, palliation of cancer and treatment of pancreatic pseudocysts. Int Surg 1996;81:1-5.

14. Tihanyi TF, Morvay K, Nehéz L, Winternitz T, Rusz Z, Flautner LE. Laparoscopic distal resection of the pancreas with the preservation of the spleen. Acta Chir Hung 1997;36:359-61.

15. Vezakis A, Davides D, Larvin M, McMahon MJ. Laparoscopic surgery combined with preservation of the spleen for distal pancreatic tumors. Surg Endosc 1999;13:26-9. 